\title{
Bifurkationsanalyse eines LC Tank VCOs unter Berücksichtigung der variablen Kapazität
}

\author{
J.-K. Bremer, C. Zorn, and W. Mathis \\ Institut für Theoretische Elektrotechnik, Leibniz Universität Hannover, Appelstr. 9A, 30167 Hannover, Deutschland
}

\begin{abstract}
Zusammenfassung. In dieser Arbeit präsentieren wir einen neuartigen Ansatz für den systematischen Entwurf von integrierten LC Tank VCO-Schaltungen basierend auf dem Andronov Hopf Theorem und der Störungstheorie. Der Ansatz ermöglicht es, eine Abschätzung des resultierenden Abstimmbereichs, eine Stabilitätsanalyse und eine Berechnung der Amplitude des VCOs im Vorfeld des eigentlichen Entwurfs durchzuführen. Des Weiteren erlaubt die vorgestellte Methode eine Optimierung des VCOs hinsichtlich der in den Spezifikationen geforderte Amplitude und eine Minimierung der höheren Harmonischen. Mit Hilfe eines ladungsbasierten MOS-Modells ist es möglich die spannungsabhängige Kapazität der Varaktortransistoren durch einen analytischen Ausdruck zu beschreiben. Auf Basis dieses analytischen Ausdrucks wird die amplitudenabhängige Großsignalkapazität des VCOs in Abhängigkeit von Designparametern und der Tuningspannung modelliert. Die Gültigkeit der vorgestellte Entwurfsmethode wird anhand eines Beispielentwurfes eines 2.4 GHz VCO unter Verwendung einer $0.25 \mu \mathrm{m}$ HF-CMOS Technologie verifiziert.
\end{abstract}

\section{Einleitung}

Durch steigende Frequenzen und immer komplexer werdende Modulationsverfahren wachsen die Anforderungen an die einzelnen Komponenten moderner HF Kommunikationssysteme. Dies hat zur Folge, dass die Spezifikationen die eine VCO, als einer der Basisblöcke moderner Kommunikatiossysteme, erfüllen muss immer schwerer zu realisieren sind. Im einzelnen sind dies die Realisierung der steigenden Frequenzen, ein großer Abstimmbereich aufgrund der steigenden Verbreitung von Breitbandapplikationen, eine niedrige Leistungsaufnahme und geringes Phasenrauschen. Des Weiteren muss der VCO hinsichtlich einer maximalen Amplitude optimiert werden, um die nachfolgende Mischerstufe

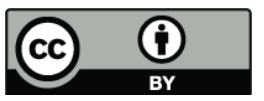

Correspondence to: J.-K. Bremer (bremer@tet.uni-hannover) zu treiben. Bei dem Entwurfsprozess von integrierten Oszillatorschaltungen handelt es sich um eine sehr komplexe Aufgabe, die stark erfahrungsbasiert ist. Deshalb wird ein systematischer Entwurfsprozess benötigt, der es ermöglicht vor der eigentlichen Schaltungssimulation mit einem Schaltungssimulator eine Abschätzung aller Designparameter durchzuführen. Basierend auf vorausgegangenen Arbeiten zur nichtlinearen Modellierung von Oszillatorschaltungen mit Hilfe des Andronov-Hopf Theorems (Mathis, 2005; Prochaska, 2005; Bremer, 2008) präsentieren wir in dieser Arbeit einen neuartigen Ansatz für einen systematischen nichtlinearen Entwurfsprozess für spannungsgesteuerte Oszillatorschaltungen. Zur exakten Modellierung der sich im VCO einstellenden effektiven Grosssignalkapazität wurden in der Vergangenheit mehrere Ansätze veröffentlicht (Tiebout, 2001; Bunch, 2003; Abidi, 2003). Unser Ansatz ermöglicht es auf Basis des ladungsbasierten EKV MOS-Modells (Enz, 1995) die spannungsabhängige Kapazität eines MOSTransistors in schwacher und starker Inversion zu beschreiben. Mit diesem Modell ist es möglich eine Abschätzung der Varaktorabmessungen durchzuführen und den Abstimmbereich des VCOs im Vorfeld des Designs zu simulieren. Auf Basis dieses Kapazitätsmodells und der nichtlinearen Modellierung der einzelnen Schaltungskomponenten ist es möglich den VCO durch ein Differentialgleichungssystem zweiter Ordnung zu beschreiben, dass nur noch von Prozessparametern und Designparametern abhängig ist. Mit Hilfe der Andronov-Hopf Bifurkationsanalyse wird das entstandene nichtlineare dynamische System auf die Existenz eines stabilen Grenzzykluses untersucht und so die Designparameter bestimmt, für die der VCO stabil schwingt. Mit Hilfe der Störungsrechnung ist es nun möglich, ausgehend von dem nichtlinearen dynamischen System das einen stabilen Grenzzyklus besitzt, analytische Ausdrücke für die Frequenz und die Amplitude des VCOs herzuleiten. Der vorgestellte Ansatz zum systematischen Entwurf von spannungsgesteuerten Oszillatorschaltungen ist generell auf alle VCO Architekturen anwendbar. Aufgrund der hohen Verbreitung der LC Tank VCO Architektur wird der Ansatz in dieser Arbeit exemplarisch auf diese angewendet. 


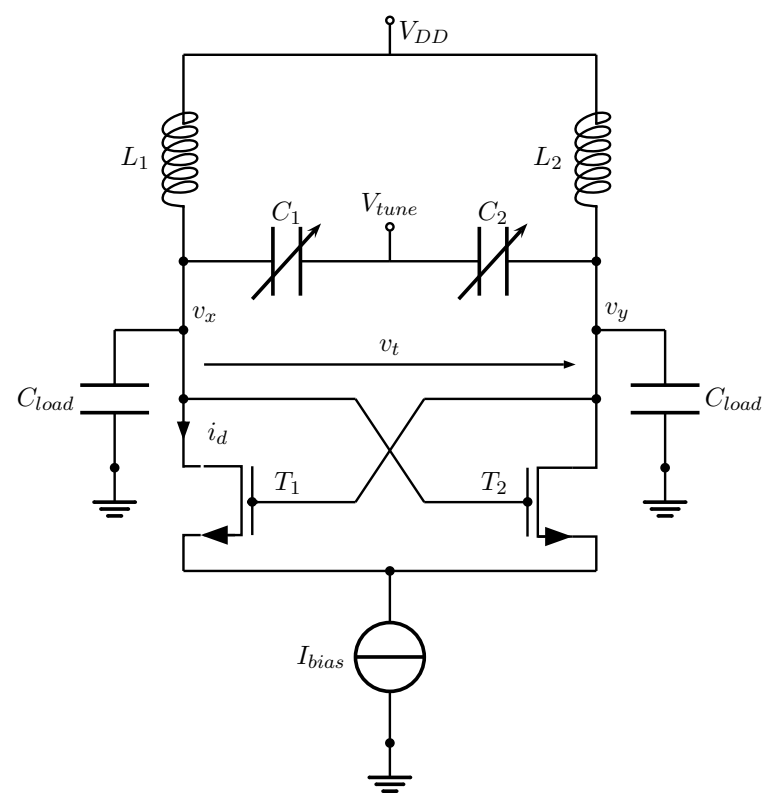

Abbildung 1. LC Tank VCO-Schaltung.

Die Arbeit ist folgendermaßen gegliedert. Im zweiten Abschnitt wird ausgehend von der LC Tank VCO Architektur die nichtlineare Modellierung der einzelnen Komponenten aufgezeigt. Der dritte Abschnitt beschäftigt sich mit dem von uns entwickelten Ansatz zur Modellierung der effektiven Grosssignalkapazität im VCO in Abhängigkeit der Tankamplitude. Im vierten Abschnitt wird ausgehend von den Modellen der einzelnen Komponenten aus Abschnitt zwei ein äquivalentes Ersatzschaltbild für die VCO-Architektur eingeführt. In Abschnitt fünf wird das nichtlineare dynamische System in Abhängigkeit der Designparameter für einen LC Tank VCO aufgestellt. Abschnitt sechs zeigt die Anwendung der Andronov-Hopf Bifurkationsanalyse auf das nichtlineare dynamische System, gefolgt von der anschließenden Störungsrechnung, um die Amplitude und die Frequenz im Bifurkationspunkt zu bestimmen. Abschließend wird in Abschnitt sieben ein Beispielentwurf für einen $2.4 \mathrm{GHz} \mathrm{VCO}$ auf Basis des vorgestellten Entwurfsprozesses durchgeführt und die theoretischen Ergebnisse mit Cadence Simulationsergebnissen verglichen.

\section{VCO Modellierung}

Ausgehend von der in Abb. 1 gezeigten stromgespeisten NMOS-only LC Tank VCO Topologie wird in diesem Abschnitt die nichtlineare Modellierung der einzelnen Schaltungskomponenten analysiert. Die Schaltung besteht aus zwei symmetrischen integrierten Spulen, aus zwei in Reihe geschalteten MOS-Varaktoren, dem kreuzgekoppelten Transistorpaar Widerstands und aus einer Stromquelle im Fusspunkt.

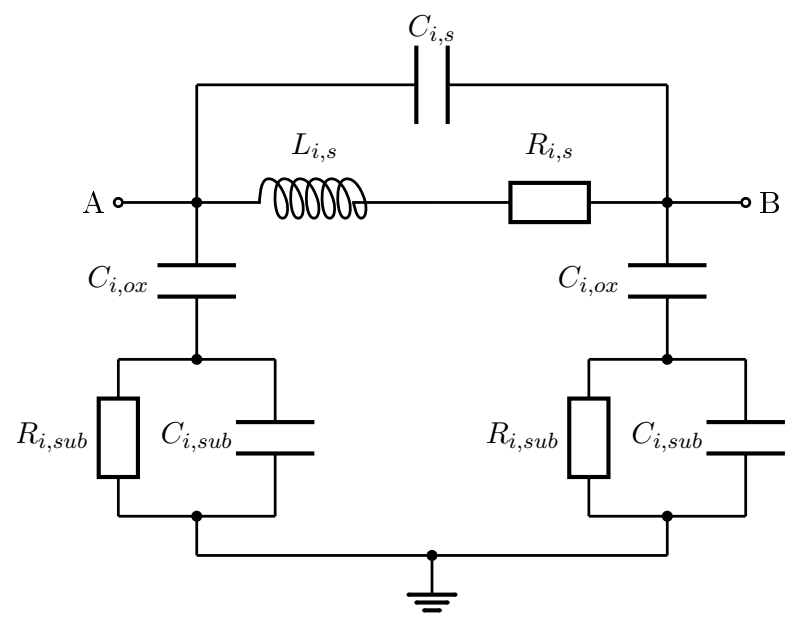

Abbildung 2. Spulenmodell aus konzentrierten Bauelementen.

\subsection{Spulenmodell}

In Standard-CMOS-Bibliotheken sind integrierte Spulen selten als Standardzellen enthalten, wie es z.B. bei Transistoren üblich ist. Deshalb wird ein Schaltungsmodell benötigt, dass über eine weiten Bereich von Layoutabmessungen und Prozessparametern skalierbar ist. Der Schlüssel zu einem physikalischen exakten Modell der integrierten Spulen ist es, die parasitären Widerstände und Kapazitäten zu berücksichtigen. Ein typisches Spulenmodell aus konzentrierten Bauelementen (Yue, 2000) ist in Abb. 2 dargestellt.

$R_{i, s}$ repräsentiert den ohmschen Widerstand der Spule der sich aus der endlichen Leitfähigkeit des verwendeten Metalls und den auftretenden Wirbelstromeffekten ergibt. $C_{i, s}$ modelliert die kapazitive Kopplung zwischen den beiden An-

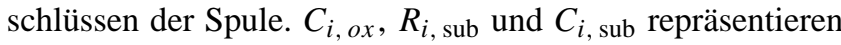
die parasitären Substrateffekte. Das Spulenmodell ist aufgrund der zusätzlichen Serienkapazität $C_{o x}$ für eine einfache Schaltungsanalyse zu kompliziert. Es ist daher sinnvoll, dass in Abb. 3 dargestellte vereinfachte Modell zu verwenden. Die frequenzabhängigen Ersatzgrößen $R_{i, \text { sup, } p}$ und $C_{i \text {, sup, } p}$ können durch die folgenden Ausdrücke berechnet werden (Yue, 1998)

$R_{i, \mathrm{sup}, p}=\frac{1+\left(\omega R_{i, \mathrm{sub}}\left(C_{i, \mathrm{sub}}+C_{i, o x}\right)\right)^{2}}{\omega^{2} R_{i, \mathrm{sub}} C_{i, o x}^{2}}$

$C_{i, \text { sup }, p}=C_{i, o x} \frac{1+\omega^{2} R_{i, \text { sub }}^{2} C_{i, \text { sup }}\left(C_{i, \text { sup }}+C_{i, o x}\right)}{1+\left(\omega R_{i, \text { sub }}\left(C_{i, \text { sub }}+C_{i, o x}\right)\right)^{2}}$.

Ersetzt man den Serienwiderstand $R_{i, s}$ durch einen äquivalenten Parallelwiderstand $R_{i, p}$ und nutzt die Symmetrie der VCO Topologie (Abb. 1) aus, so ist es möglich das Spulenersatzschaltbild in Abb. 4 zu erhalten, welches ausschließlich aus parallelgeschalteten Schaltungselementen besteht. 


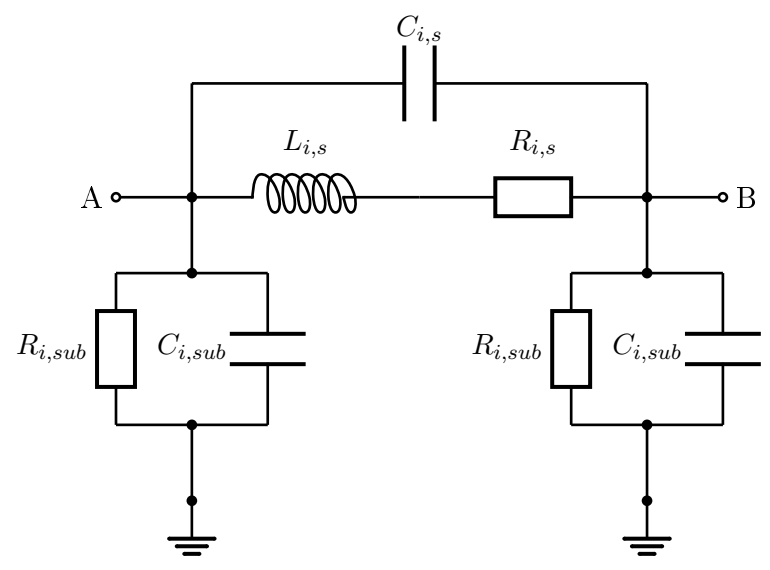

Abbildung 3. Vereinfachtes Spulenmodell.

Nachteil der vereinfachten Spulenmodelle ist, dass die berechnet Schaltungselemente nur für einen kleinen Frequenzbereich Gültigkeit besitzen.

\subsection{MOS-Varaktor Modellierung}

Unser Modellierungsansatz beschränkt sich auf InversionMode MOS Varaktoren, da diese im Gegensatz zu Akkumulation-Mode Varaktoren aus Standard-MOSFETZellen hergestellt werden. Im Falle eines NMOS Transistors werden der Sourceanschluss und der Drainanschluss kurzgeschlossen und der Bulkanschluss mit Masse verbunden. Dies führt dazu, dass der resultierende Inversion-Mode Varaktor nur noch im Verarmungsbereich betrieben wird und den Akkumulationsbereich nicht mehr erreicht. Wir modellieren den Inversion-Mode Varaktor als eine Parallelschaltung eines ohmschen Widerstands und einer nichtlinearen spannungsabhängigen Kapazität $C_{v}\left(V_{G S}, V_{\text {tune }}\right)$ (siehe Abb. 5).

Der Serienwiderstand eines Inversion-Mode MOS Varaktors $R_{v, s}$ setzt sich aus dem Gatewiderstand, den Kontaktwiderständen zum Polysilizium und den Diffusionsgebieten und dem Widerstand des invertierten Kanals zusammen. Er lässt sich näherungsweise durch den folgenden Ausdruck bestimmen (Bunch, 2003)

$R_{v, s}=\frac{1}{12} \cdot \frac{1}{N} \cdot\left(R_{c h, \square} \cdot \frac{L_{v}}{W_{v}}+R_{\mathrm{poly}, \square} \cdot \frac{W_{v}}{L_{v}}\right)$.

Dabei bezeichnen $R_{c h}, \square$ den Schichtwiderstand der Inversionsschicht, $R_{\text {poly, }} \square$ den Polysilizium Schichtwiderstand und $N$ die Anzahl der Gatefinger. Durch eine anschlieBende Reihen-Parallelwandlung lässt sich der Varaktorserienwiderstand $R_{v, s}$ in den äquivalenten Parallelwiderstand $R_{v, p}$ umrechnen. Um einen analytischen Ausdruck für die Kapazitäts-Spannungs-Charakteristik $C_{v}\left(V_{G S}, V_{\text {tune }}\right)$ eines Inversion-Mode MOS Varaktors $\mathrm{zu}$ erhalten, ist eine in schwacher und starker Inversion kontinuierliche Beschreibung der MOS-Kapazität erforderlich. Die häufig verwendeten, nur abschnittsweise gültigen MOS-Modelle sind für

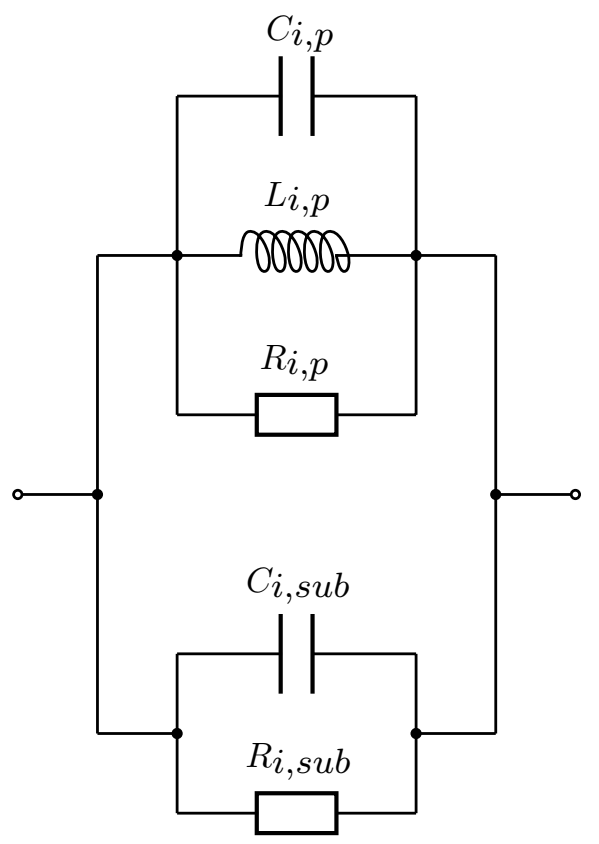

Abbildung 4. Spulenmodell aus parallelgeschalteten Schaltungselementen.

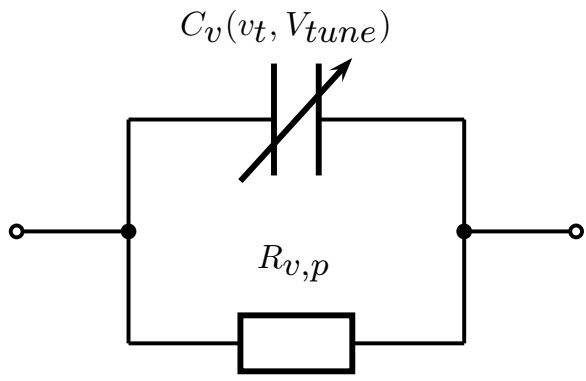

Abbildung 5. Varaktormodell.

diesen Zweck nicht brauchbar. Das EKV Transistormodell liefert eine gut geeignete analytische Interpolation für die MOS Kapazitäten in den geforderten Bereichen. Die einzelnen interpolierten intrinsischen MOS-Kapazitäten sind gegeben durch (Enz, 1995)

$$
\begin{aligned}
& C_{g s}=C_{o x} \cdot\left[\frac{1}{c_{g s s}\left(i_{f}, i_{r}\right)}+\frac{1}{c_{g s w}\left(i_{f}\right)}\right]^{-1} \\
& C_{g d}=C_{o x} \cdot\left[\frac{1}{c_{g s s}\left(i_{r}, i_{f}\right)}+\frac{1}{c_{g s w}\left(i_{r}\right)}\right]^{-1} \\
& C_{g b}=C_{o x} \cdot\left(\frac{n-1}{n}\right) \cdot\left[1-\frac{c_{g b s}\left(i_{f}, i_{r}\right) \cdot c_{g b w}\left(i_{f}, i_{r}\right)}{c_{g b s}\left(i_{f}, i_{r}\right)+c_{g b w}\left(i_{f}, i_{r}\right)}\right] \\
& C_{b s}=(n-1) \cdot C_{g s}
\end{aligned}
$$




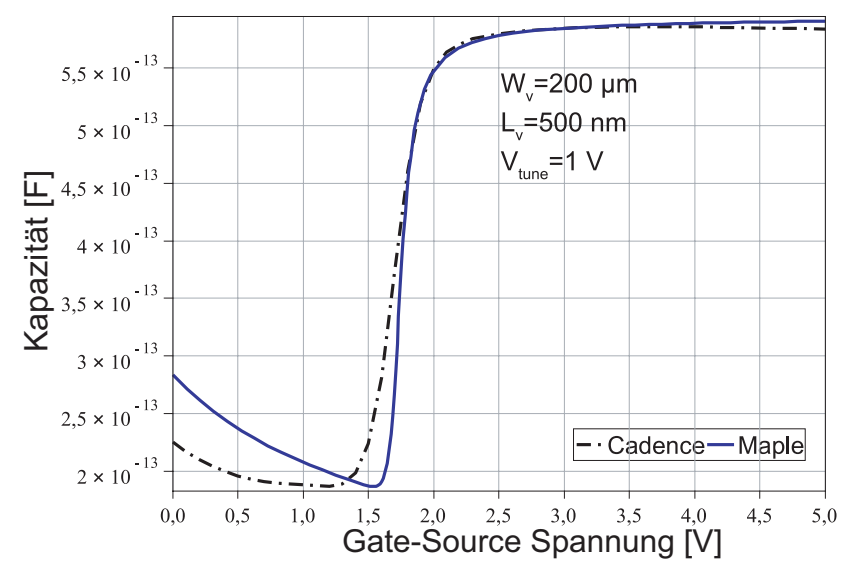

Abbildung 6. CV-Charakteristik eines NMOS Inversion-Mode Varaktors.

$C_{b d}=(n-1) \cdot C_{g d}$

$i_{f}$ und $i_{r}$ bezeichnen die Vorwärts- und Rückwärtskomponente des auf die Temperaturspannung normierten Drainstroms und $n$ entspricht dem so genannten "slope factor". Die Ausdrücke $c_{g s s}, c_{g s w}, c_{g b s}$ und $c_{g b w}$ bezeichnen die intrinsischen Kapazitäten in starker beziehungsweise schwacher Inversion (siehe Enz, 1995).

Fasst man die fünf intrinsischen Kapazitäten zusammen

$C_{v}\left(v_{t}, V_{\text {tune }}\right)=C_{g s}+C_{g d}+C_{g b}+C_{b s}+C_{b d}$

ist es möglich, eine gute Approximation der CVCharakteristik eines Inversion-Mode Varaktors zu erhalten. Ein Vergleich der analytischen Näherung mit Spectre (Cadence DF) Simulationsergebnissen ist in Abb. 6 dargestellt.

\subsection{Differentielles Transistorpaar}

Die nichtlineare Strom-Spannungs-Charakteristik eines kreuzgekoppelten MOS-Transistorpaares weist in einem gewissen Bereich eine negative Steigung auf und kann durch den in (Buonomo, 2004) veröffentlichten Zusammenhang effektiv beschrieben werden

$i_{d}\left(v_{t}\right)= \begin{cases}\frac{I_{\text {bias }}}{2}, & v_{t}<-\frac{v_{n}}{\sqrt{2}} \\ -\frac{I_{\text {bias }} v_{t}}{v_{n}} \sqrt{1-\left(\frac{v_{t}}{v_{n}}\right)^{2}}, & -\frac{v_{n}}{\sqrt{2}} \leq v_{t} \leq \frac{v_{n}}{\sqrt{2}} \\ \frac{I_{\text {bias }}}{2}, & v_{t}>\frac{v_{n}}{\sqrt{2}}\end{cases}$

mit

$v_{n}=2 \sqrt{\frac{I_{\text {bias }} L_{n}}{\mu_{n} C_{o x} W_{n}}}$.
Um eine möglichst genaue Abschätzung der resultierenden Gesamtkapazität des VCOs zu erhalten, müssen die parasitären Kapazitäten des differentiellen Transistorpaares mitberücksichtigt werden. Eine Näherung für die parasitären Kapazitäten eines kreuzgekoppelten NMOS-Paares ist in Hershenson (1999) zu finden

$C_{\mathrm{NMOS}, \text { pair }}=2 C_{g d, n}+\frac{1}{2} C_{g s, n}+\frac{1}{2} C_{d b, n}$.

Eine Berechnung der benötigten Transistorkapazitäten ist mit Hilfe der Formeln (4), (5) und (8) möglich.

\section{Modellierung der Großsignalkapazität}

In der Standard LC Tank VCO Topologie (vgl. Abb. 1) liegt die Ausgangsamplitude des VCOs direkt über den Gateanschlüssen der verwendeten MOS Varaktoren. Die differentielle Amplitude des Ausgangssignals der meisten VCOs entspricht fast zweimal dem Maximum der zur Verfügung stehenden Spannungsversorgung. Dies bedeutet, dass sich die einstellende effektive Kapazität der Varaktoren periodisch mit der Amplitude des Ausgangssignal $v_{t}(t)$ ändert. Daraus folgt, dass zur Berechnung der sich einstellenden Kapazität eine Großsignalanalyse erforderlich ist. Die Abhängigkeit der Varaktorkapazität von der Tankamplitude hat zwei Auswirkungen. Eine ist, dass kleine Amplitudenschwankungen z.B. aufgrund von Rauschen die effektive Kapazität modulieren und damit die Ausgangsfrequenz des VCOs verändern. Dieser Vorgang wird AM-zu-FM Konvertierung genannt (für Details siehe Abidi, 2003). Die andere ist, dass die während einer Periode variierende Varaktorkapazität zu einer Verzerrung des sinusförmigen Ausgangssignals führt. In der Vergangenheit wurden einige Ansätze veröffentlicht, die mit Hilfe einer Fourieranalyse die Großsignalkapazität für VCOSchaltungen bestimmen (Tiebout, 2001; Bunch, 2003; Abidi, 2003). Mit Hilfe des in Abschnitt 2.2 gezeigten Kapazitätsmodells für die Varaktoren, ist es möglich einen analytischen Ausdruck für die effektive Großsignalkapazität in Abhängigkeit der Tankamplitude $v_{t}(t)$ aufzustellen, der zu jedem Zeitpunkt der Oszillation gültig ist. Aufgrund des symmetrischen Aufbaus der VCO-Schaltung (vgl. Abb. 1) ist es eine gültige Annahme die beiden MOS-Varaktortransistoren als vollständig symmetrisch anzunehmen. Damit folgt für das differentielle Ausgangssignal des VCOs

$v_{t}(t)=v_{x}(t)-v_{y}(t)=\frac{v_{t}(t)}{2}-\frac{-v_{t}(t)}{2}$.

Die effektive Gesamtvaraktorkapazität setzt sich aus der Reihenschaltung der beiden spannungsabhängigen Kapazitäten der einzelnen Varaktortransistoren $C_{1}\left(v_{t}, V_{\text {tune }}\right)$ und $C_{2}\left(v_{t}, V_{\text {tune }}\right)$ zusammen, die sowohl von der Tuningspannung, als auch von der Ausgangsamplitude des VCOs abhängen

$C_{v, \text { eff }}\left(v_{t}, V_{\text {tune }}\right)=\frac{C_{1}\left(\frac{v_{t}}{2}, V_{\text {tune }}\right) \cdot C_{2}\left(-\frac{v_{t}}{2}, V_{\text {tune }}\right)}{C_{1}\left(\frac{v_{t}}{2}, V_{\text {tune }}\right)+C_{2}\left(-\frac{v_{t}}{2}, V_{\text {tune }}\right)}$. 


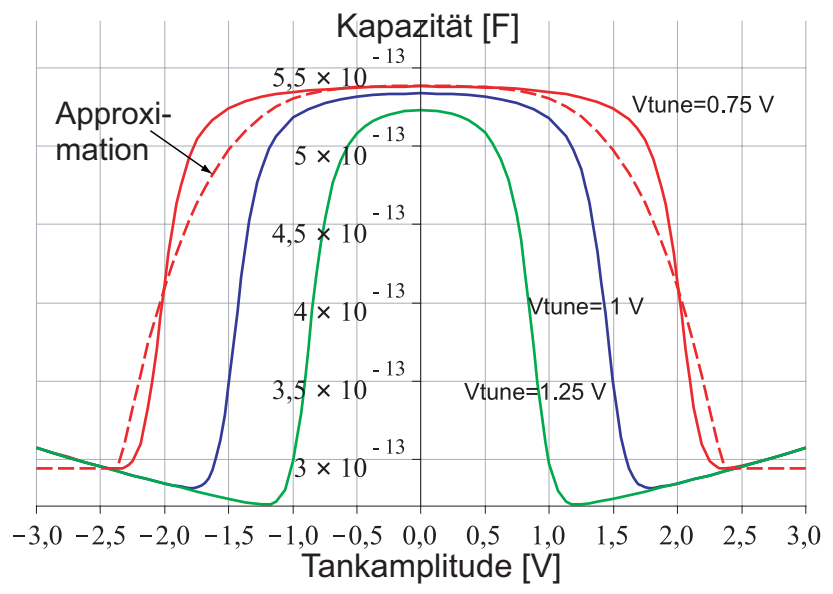

Abbildung 7. Effektive Gesamtvaraktorkapazität eines InversionMode Varaktors mit einer Transistorweite von $W_{v}=400 \mu \mathrm{m}$.

Abbildung 7 zeigt die mit Hilfe von (14) berechnete effektive Gesamtvaraktorkapazität für einen Inversion-Mode Varaktor mit einer Transistorweite von $400 \mu \mathrm{m}$ für verschieden Tuningspannungen. Für eine Tankamplitude von $v_{t}=0 \mathrm{~V}$ besitzen beide Varaktortransistoren die gleiche effektive Kapazität $C_{1}(0)=C_{2}(0)$. An diesem Punkt entspricht die effektive Gesamtvaraktorkapazität genau der halben effektiven Kapazität eines Varaktortransistors (siehe Abb. 7)

$C_{v, \text { eff }}\left(v_{t}=0, V_{\text {tune }}\right)=C_{v 0}\left(V_{\text {tune }}\right)$.

Der resultierende analytische Ausdruck für die effektive Varaktorkapazität ist zu komplex um ihn in der Andronov-Hopf Bifurkationsanalyse zu verwenden. Die folgende stückweise nichtlineare Funktion für $C_{v}$, eff $\left(v_{t}, V_{\text {tune }}\right)$

$= \begin{cases}C_{v, \text { eff }}\left(v_{t, \text { turn }}, V_{\text {tune }}\right), & v_{t}<-v_{t, \text { turn }} \\ A\left(V_{\text {tune }}\right) \cdot v_{t}^{4}+\frac{C_{v 0}\left(V_{\text {tune }}\right)}{2}, & -v_{t, \text { turn }}<v_{t}<v_{t, \text { turn }} \\ C_{v, \text { eff }}\left(v_{t, \text { turn }}, V_{\text {tune }}\right), & v_{t}>v_{t, \text { turn }}\end{cases}$

ist aufgrund der guten Übereinstimmung (siehe Abb. 7) und der viel geringere Komplexität in erster Näherung gut geeignet, in der Andronov-Hopf Bifurkationsanalyse verwendet zu werden. Die Spannung $v_{t}$, turn bezeichnet die Spannung, bei der die Funktion (16) das lokale Minimum besitzt. Der Parameter $A\left(V_{\text {tune }}\right)$ ist abhängig von der Tuningspannung und kann folgendermaßen bestimmt werden

$A\left(V_{\text {tune }}\right)=\frac{C_{v, \text { eff }}\left(v_{t}=v_{t, \text { turn }}\right)}{v_{t, \text { turn }}^{4}+\frac{C_{v 0}\left(V_{\text {tune }}\right)}{2}}$.

\section{VCO Ersatzschaltbild}

Unter Ausnutzung aller Schaltungssymmetrien und der in Abschnitt 2 vorgestellten nichtlinearen Modellierung, lässt

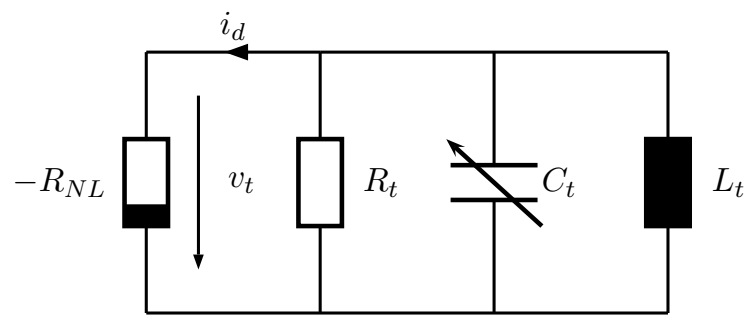

Abbildung 8. Äquivalentes VCO Ersatzschaltbild.

sich die VCO Schaltung in Abb. 1 durch das in Abb. 8 gezeigte Ersatzschaltbild ersetzen. Die einzelnen Elemente des Ersatzschaltbildes ergeben sich zu

$$
\begin{aligned}
C_{t}= & C_{v, \text { eff }}\left(v_{t}, V_{\text {tune }}\right)+\frac{1}{2}\left(C_{\text {load }}+C_{i, p}+C_{i, \text { sub }, p}\right) \\
& +C_{\text {NMOS }, \text { pair }} \\
= & C_{v, \text { eff }}\left(v_{t}, V_{\text {tune }}\right)+C_{\text {par }} \\
L_{t}= & 2 L_{i, p} \\
R_{t}= & 2\left[\frac{1}{R_{v, p}}+\frac{1}{R_{i, p}}+\frac{1}{R_{i, \text { sub }, p}}+\frac{1}{R_{0}}\right]^{-1} .
\end{aligned}
$$

$R_{0}$ entspricht dem Ausgangswiderstand des differentiellen Transistorpaares. Der negative Widerstand $R_{N L}$ wird durch den Ausdruck (10) beschrieben. Aufgrund der nichtlinearen Modellierung der spannungsgesteuerten Kapazität und des differentiellen Transistorpaares ist dieses einfache Ersatzschaltbild sehr gut geeignet das qualitative Verhalten einer LC Tank VCO-Schaltung zu beschreiben.

\section{Nichtlineares dynamisches System}

Im nachfolgenden Abschnitt wird auf Grundlage der in Abschnitt 2 vorgestellten Modellierung ein nichtlineares dynamisches System zweiter Ordnung für einen LC Tank VCO aufgestellt. Im ersten Schritt werden hierfür die Netzwerkgleichungen für das VCO-Ersatzschaltbild (siehe Abb. 8) hergeleitet. Mit Hilfe des nichtlinearen Ausdrucks für den Strom (10) und der effektiven Tankkapazität (16) erhält man die folgenden Zustandsgleichungen für die LC Tank VCOSchaltung

$$
\begin{aligned}
& \frac{d v_{t}}{d t}=-\frac{v_{t}}{R_{t} \cdot\left[A\left(V_{\text {tune }}\right) \cdot v_{t}^{4}+C_{v 0}\left(V_{\text {tune }}\right)+C_{\text {par }}\right]} \\
& -\frac{i_{L}}{A\left(V_{\text {tune }}\right) \cdot v_{t}^{4}+C_{v 0}\left(V_{\text {tune }}\right)+C_{\text {par }}} \\
& -\frac{-I_{\text {bias }} \cdot \frac{v_{t}}{v_{n}} \cdot \sqrt{1-\frac{v_{t}^{2}}{v_{n}^{2}}}}{A\left(V_{\text {tune }}\right) \cdot v_{t}^{4}+C_{v 0}\left(V_{\text {tune }}\right)+C_{\text {par }}}
\end{aligned}
$$


und

$\frac{d i L}{d t}=\frac{v_{t}}{L_{t}}$

mit

$v_{n}=2 \sqrt{\frac{I_{\mathrm{bias}} L_{n}}{\mu_{n} C_{o x} W_{n}}}$.

Nach einer Aufspaltung von $i_{d}\left(v_{t}\right)$ and $C_{t}\left(v_{t}, V_{\text {tune }}\right)$ in linearen und nichtlinearen Teil erhält man ein dynamisches System für einen LC Tank VCO, dass nur noch von Design- und Prozessparametern abhängig ist

$$
\begin{aligned}
& \left(\begin{array}{c}
\frac{d v_{t}}{d t} \\
\frac{d i_{L}}{d t}
\end{array}\right)=\left(\begin{array}{cc}
\frac{1}{C_{0}\left(V_{\text {tune }}\right)}\left[\frac{I_{\text {bias }}}{v_{n}}-\frac{1}{R_{t}}\right] & -\frac{1}{C_{0}\left(V_{\text {tune }}\right)} \\
\frac{1}{L_{t}} & 0
\end{array}\right) \\
& \left.\times\left(\begin{array}{c}
v_{t} \\
i_{L}
\end{array}\right)+\left(\begin{array}{c}
-\frac{v_{t}^{3} I_{\text {bias }} C_{0}\left(V_{\text {tune }}\right)}{2 v_{n}^{3}} \\
+A\left(V_{\text {tune }}\right) v_{t}^{4} i_{L} \\
+\frac{A\left(V_{\text {tune }}\right) v_{t}^{5}}{R_{t}} \\
-\frac{A\left(V_{\text {tune }}\right) v_{t}^{5} I_{\text {bias }}}{\left.v_{n}\left(V_{\text {tune }}\right)\right]^{2}} \\
+\frac{A\left(V_{\text {tune }}\right) v_{t}^{7} I_{\text {bias }}}{2 v_{n}^{3}}
\end{array}\right)\right),
\end{aligned}
$$

mit

$C_{0}\left(V_{\text {tune }}\right)=C_{v 0}\left(V_{\text {tune }}\right)+C_{\text {par }}$.

Dies entspricht einem Differentialgleichungssystem folgender Form

$\dot{\mathbf{x}}=\mathbf{A x}+\tilde{\mathbf{f}}(\mathbf{x})$

mit dem linearen Anteil $\mathbf{x}$ und dem nichtlinearen Anteil $\tilde{\mathbf{f}}(\mathbf{x})$.

\section{Bifurkationsanalyse}

Die Andronov-Hopf Bifurkationsanalyse ist eine effiziente Methode für die Untersuchung von periodischen Lösungen autonomer einparametriger nichtlinearer gewöhnlicher Differentialgleichungen erster Ordnung (vgl. Kazarinoff, 1981; Kuznetsov, 2004 und Guckenheimer, 1983). Der Satz erlaubt es, qualitative Informationen über die Frequenz und die Amplitude des untersuchten Systems zu erhalten, sowie eine Aussage über die Stabilität der periodischen Lösung zu machen. Dieses Kapitel gibt einen kurze Einführung in das Theorem, gefolgt von der Anwendung des Theorems auf unser System. Ein nichtlineares dynamisches System der folgenden Form ist der Ausgangspunkt für die Andronov-Hopf Bifurkation:

$\dot{\mathbf{x}}=\mathbf{f}(\mathbf{x}, \mu) \quad \mathbf{f}: \mathbb{R}^{n} \rightarrow \mathbb{R}^{n} \quad \mathbf{x} \in \mathbb{R}^{n} ; \quad \mu \in \mathbb{R}^{1}$
In der Funktion $\mathbf{f}(\mathbf{x}, \mu)$, repräsentiert $\mu$ einen frei wählbaren Netzwerkparameter oder eine Kombination von Netzwerkparametern, die zur späteren Optimierung des Systems hinsichtlich der geforderten Spezifikationen zur Verfügung stehen. $f\left(\mathbf{x}=x_{C}, \mu_{C}\right)=\mathbf{0}$ sei ein Gleichgewichtspunkt des Systems (27) für den Parameterwert $\mu=\mu_{C}$. Wenn die folgenden Bedingungen

\section{Andronov-Hopf Theorem}

C1. Die Jacobimatrix $\left.\mathbf{J}_{f}(\mathbf{x}, \mu)\right|_{\mu=\mu_{C}} ^{\mathbf{x}=\mathbf{x}_{\mathbf{C}}}$ besitzt ein konjugiert komplexes Eigenwertpaar $\lambda_{1,2}(\mu)=\alpha(\mu) \pm j \omega(\mu)$ mit den zusätzlichen Bedingungen:

$$
\left.\omega(\mu)\right|_{\mu=\mu_{C}}=\omega_{C}>0 \text { und }\left.\quad \alpha(\mu)\right|_{\mu=\mu_{C}}=0
$$

C2. Alle weiteren Eigenwerte besitzen einen negativen Realteil

C3. Die Transversalitätsbedingung $\partial \alpha(\mu) /\left.\partial \mu\right|_{\mu=\mu_{C}} \neq 0$

C4. Der erste Lyapunovkoeffizient $l_{1}$ ist negativ, bedeutet der Gleichgewichtspunkt ist asymptotisch stabil für $\mu=\mu_{C}$

erfüllt sind, ist garantiert, dass ein Bifurkationsparameter $\mu$ existiert, dessen Variation zu einem stabilen Grenzzyklus führt. Eine Vergrößerung des Bifurkationsparameters führt zu einer Vergrößerung des Durchmessers des Grenzzyklus und folglich zu einem Anstieg der Oszillatoramplitude (vgl. Guckenheimer, 1983 und Mathis, 2005). Als nächstes wird die Andronov-Hopf Bifurkationanalyse auf unser System angewendet. Zuerst muss der Bifurkationsparameter des Systems (24) ausgewählt werden. Der Parameter muss es ermöglichen den Bifurkationspunkt einzustellen, darf darüber hinaus aber möglichst keinen Einfluss auf die Frequenz des Systems haben. Die Weite des kreuzgekoppelten Transistorpaares in (23) eignet sich sehr gut als Bifurkationsparameter, da eine Variation derselbigen es ermöglicht eine stabile Schwingung zu erzeugen und gleichzeitig fast keinen Einfluss auf die sich einstellende Frequenz des Systems hat.

Die Eigenwerte der Jakobimatrix lassen sich einfach bestimmen

$\lambda_{1,2}=+\frac{\alpha}{2} \pm \sqrt{\left(\frac{\alpha}{2}\right)^{2}-\frac{1}{C_{0}\left(V_{\text {tune }}\right) L_{t}}}$,

mit

$\alpha=-\frac{1}{C_{0}\left(V_{\text {tune }}\right)}\left[-\frac{1}{R_{t}}+\frac{I_{\text {bias }} \sqrt{\mu_{n} C_{o x} W_{n}}}{2 \sqrt{I_{\text {bias }} L_{n}}}-\operatorname{frac} 1 R_{t}\right]$.

Für das Differentialgleichungssystem des LC Tank VCOs ist die Bedingung (C1.) für eine Weite von

$W_{n}=\frac{4 L_{n}}{\mu_{n} C_{o x} I_{\mathrm{bias}} R_{t}^{2}}$ 
erfüllt. Ausgehend von diesem Ergebnis lässt sich die Oszillationsfrequenz direkt bestimmen $\omega_{C}=\sqrt{1 / C_{0}\left(V_{\text {tune }}\right) L_{t}}$. Da das Differentialgleichungssystem eine Ordnung von $n=2$ besitzt ist die Bedingung (C2.) automatisch erfüllt. Die Transversalitätsbedingung (C3.) ist auch erfüllt

$$
\left.\frac{\partial \sigma(\mu)}{\partial \mu}\right|_{\mu=\mu_{C}}=\frac{I_{\text {bias }}^{2} L_{n}}{4\left(\frac{I_{\text {bias }} L_{n}}{\mu_{n} C_{o x} W_{n, C}}\right)^{3 / 2} C_{0}\left(V_{\text {tune }}\right) \mu_{n} C_{o x} W_{n, C}^{2}} .
$$

Um die letzte Bedingung (C4.) zu überprüfen muss der erste Lyapunovkoeffizient $l_{1}$ für das System bestimmt werden. Nach der in (Kuznetsov, 2004) beschriebenen Methode lässt sich der Lyapunovkoeffizient folgendermaßen bestimmen

$l_{1}(\mu)=\frac{i}{2 \omega_{0}^{2}} \operatorname{Re}\left(i g_{20} g_{11}+\omega_{0} g_{21}\right)$.

Die Bestimmung der Taylorkoeffizienten in (32) ergeben für unser System

$$
g_{20}=0, g_{11}=0, g_{21}=-\frac{3 L_{t} I_{\mathrm{bias}}}{16 C_{0}^{2}\left(\frac{I_{\mathrm{bias}} L_{n}}{\mu_{n} C_{o x} W_{n}}\right)^{3 / 2}} .
$$

Damit erhält man den Lyapunovkoeffizienten für das Differentialgleichungssystem eines LC Tank VCOs

$l_{1}(\mu)=-\frac{3}{32} \frac{L_{t} I_{\text {bias }} \omega_{C}}{\omega_{C}^{2} C_{0}\left(V_{\text {tune }}\right)^{2}\left(\frac{I_{\text {bias }} L_{n}}{\mu_{n} C_{o x} W_{n}}\right)^{3 / 2}}<0$.

Das Ergebnis (34) zeigt, dass die Bedingung (C4.) immer erfüllt ist, da es sich bei den vorkommenden Parametern ausschließlich um Schaltungsparameter handelt, die immer ein positiven Wert besitzen. Damit ist gezeigt, dass das untersuchte Differentialgleichungssystem für $\mu=\mu_{C}$ einen stabilen Grenzzyklus aufweist.

\subsection{Störungsrechnung}

Um im ersten Schritt eine Abschätzung der Amplitude des zu entwerfenden VCOs zu erhalten benutzen wir einen Ansatz aus der Störungsrechnung. Um diesen Ansatz anzuwenden wird unser DGL-System als linearer Oszillator, der von einer kleinen Nichtlinearität $\epsilon$ gestört wird aufgefasst

$$
\left(\begin{array}{c}
\frac{d i_{L}}{d t} \\
\frac{d v_{t}}{d t}
\end{array}\right)=\left(\begin{array}{cc}
0 & -\omega_{C} \\
\omega_{C} & \alpha(\mu)
\end{array}\right)\left(\begin{array}{c}
\tilde{i_{L}} \\
\tilde{v}_{t}
\end{array}\right)+\varepsilon \tilde{f}\left(\tilde{v}_{t}, \tilde{i_{L}}\right) .
$$

Als nächstes wird das System in Polarkordinaten transformiert und die folgenden Ersetzungen $v_{t}=r \sin (\theta)$ und $i_{L}=r \cos (\theta)$ durchgeführt. In dem resultierenden DGLSystem sind die beiden Zustandsgleichungen für $\cdot r$ und $\cdot \theta$ gekoppelt und es ist nicht möglich die Amplitude direkt zu bestimmen. Durch Anwendung der so genannten Mittelwertmethode Stiefel, 1978; Mathis, 1987 ist es möglich die beiden Gleichungen zu entkoppeln und die stationäre Amplitude des gemittelten Systems zu bestimmen

$$
r=\sqrt{\frac{8}{3}} \cdot \frac{\sqrt{R_{t} I_{\text {bias }}\left(R_{t} I_{\text {bias }}-v_{n}\right)} \cdot v_{n}}{R_{t} I_{\text {bias }}} .
$$

\section{Systematischer Entwurfsprozess}

In dem folgenden Abschnitt wird die Anwendung des systematischen Entwurfsprozesses, auf Basis der in den vorangegangenen Abschnitten vorgestellten Ansätze, am Beispiel eines $2.4 \mathrm{GHz} \mathrm{VCO}$, wie er z.B. für Bluetoothanwendungen zum Einsatz kommt, gezeigt. Der zugrundeliegende Halbleiterprozess ist ein $0.25 \mu \mathrm{m}$ RF CMOS Prozess der Firma IHP (SGB25V). Die geforderten Spezifikationen sind in der Tabelle in Abb. 11 zusammengefasst. Um den in den Spezifikationen geforderten Abstimmbereich zu erfüllen, wird eine Spule mit einer Serieninduktivität von $L_{i, s}=11.1 \mathrm{nH}$ ausgewählt. Im nächsten Schritt soll der Varaktor dimensioniert werden. Dies lässt sich mit Hilfe des in Abschnitt 2.2 vorgestellten spannungsabhängigen Modell für die Varaktorkapazität realisieren. Abbildung 9 zeigt eine Näherung des resultierenden Abstimmbereichs des VCOs in Abhängigkeit der Varaktortransistorweite $W_{v}$. Die Simulationsergebnisse zeigen, dass eine Weite von $W_{n}=150 \mu \mathrm{m}$ ein guter Startwert ist, um den geforderten Abstimmbereich $\mathrm{zu}$ erfüllen (vgl. Abb. 9).

Die Varaktortransistorlänge $L_{v}$ wird auf das zweifache der minimalen zulässigen Kanallänge gesetzt, um einen guten Kompromiss zwischen dem Serienwiderstand und dem Verhältnis $C_{v, \max } / C_{v, \min }$ zu erzielen (Bunch, 2003). Die Länge des differentiellen Transistorpaares $L_{n}$ wird auf die minimale Kanallänge gesetzt, um den parasitären Widerstand zu minimieren. Der Biasstrom $I_{\text {bias }}$ hingegegen wird auf den in der Spezifikation zulässigen Wert maximiert, um die Ausgangsamplitude zu maximieren und das Phasenrauschen des VCOs zu minimieren (Hershenson, 1999). Außer der Weite des differentiellen Transistorpaares $W_{n}$ sind nun alle Entwurfsparameter festgelegt. Wie in Abschnitt 6 gezeigt wurde ist garantiert, dass die Variation der Weite des differentiellen Transistorpaares $W_{n}$ zu einem stabilen Grenzzyklus führt. Mit Hilfe des Zusammenhangs (30) ist es möglich die benötigte Weite $W_{n} \mathrm{zu}$ berechnen. Man findet den Bifurkationspunkt und damit den Startpunkt einer stabilen Schwingung bei einem Parameterwert von $W_{n}=2.03 \mu \mathrm{m}$. Eine Erhöhung von $W_{n}$ führt nach (36) zu einer Vergrößerung des Durchmessers des Grenzzykluses und damit zu einer Vergrößerung der Ausgangsamplitude (siehe Abb. 9).

Die bisher bestimmten Entwurfsparameter sind noch einmal in Abb. 11 zusammengefasst. Um die theoretischen Simulationsergebnisse zu verifizieren wurde die VCO-Schaltung mit diesem Parameterset in Spectre, unter Verwendung eines BSIM 3.4 MOS Modells, simuliert. Die Simulationen ergaben, dass sich für eine Weite des differentiellen Transistorpaares von $W_{n}=3.05 \mu \mathrm{m}$ eine stabile Schwingung des VCOs 

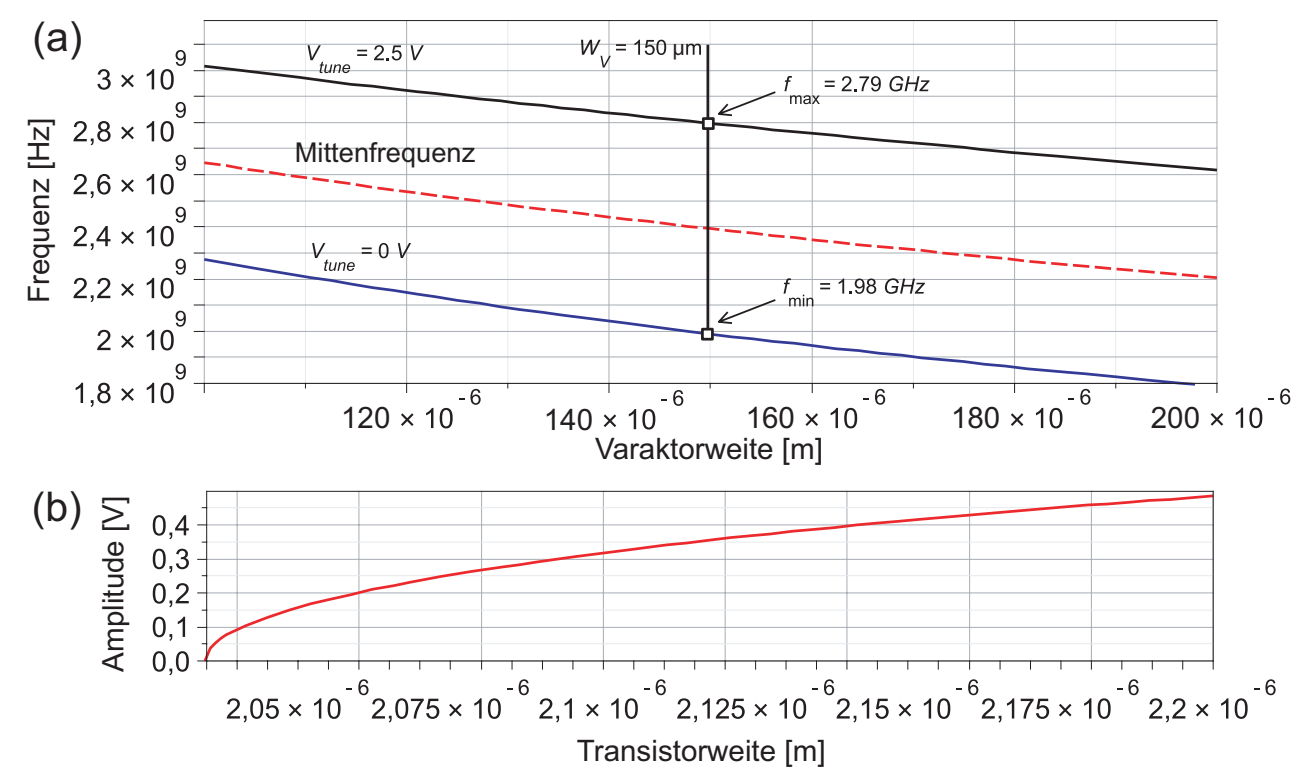

Abbildung 9. Maple: (a) Abschätzung des Abstimmbereiches, (b) Abschätzung der Amplitude.
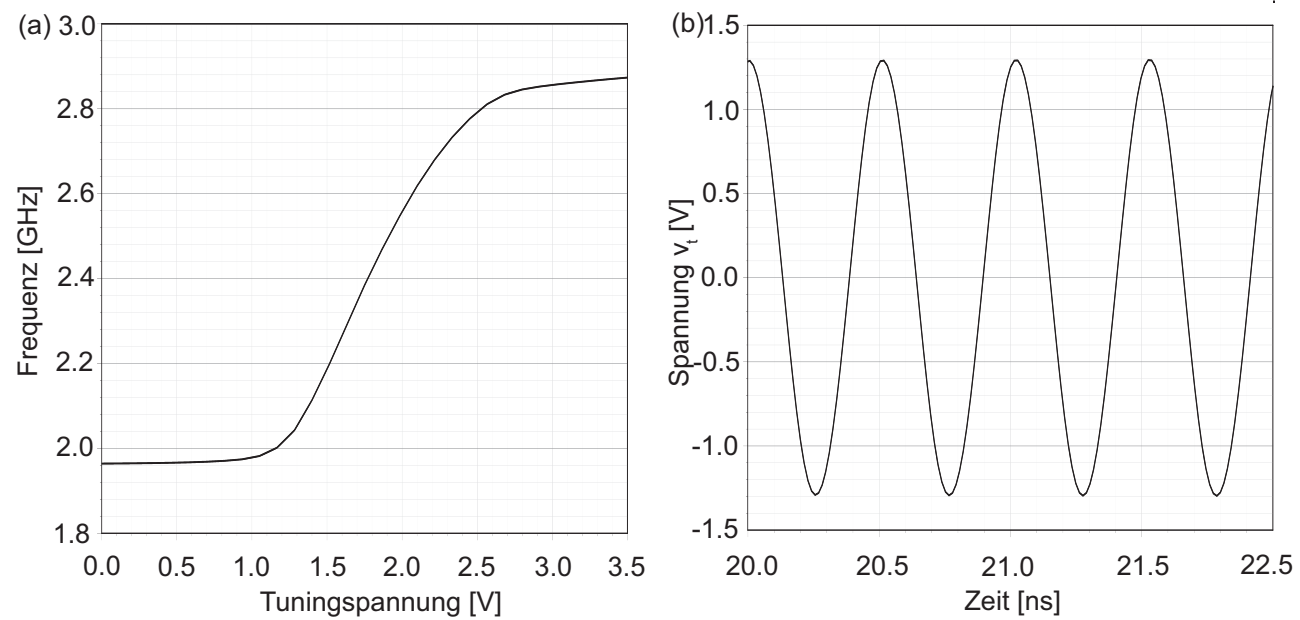

Abbildung 10. Cadence: (a) Frequenzcharakteristik, (b) Transientes Ausgangssignal.

ausbildet. Dieses Simulationsergebnis liegt sehr nahe an dem von uns berechneten Wert. Die Abweichung ist auf die unterschiedliche Komplexität der verwendeten MOS-Modelle zurückzuführen. Die Berechnung der Tankamplitude ist nur in der Nähe des Bifurkationspunktes gültig (vgl. Abb. 9). In diesem Bereich ist der Betrag der Amplitude zu klein, um die geforderte Spezifikation zu erfüllen. Aus Gleichung (36) ist bekannt, dass eine Vergrößerung der Weite $W_{n}$ zu einer Vergrößerung der Amplitude führt. Deshalb eignet sich die mit der Bifurkationsanalyse bestimmte Weite des differentiellen Tansistorpaares gut als Startpunkt für eine Parameteranalyse. Simulationen mit Spectre ergaben, dass für eine Weite von $W_{n}=4 \mu \mathrm{m}$ die Amplitude des VCOs $v_{t}=1.37 \mathrm{~V}$ beträgt. Dieser Wert erfüllt die geforderte Amplitudenspezifikation. Eine Vergrößerung der Weite über $4 \mu \mathrm{m}$ hinaus führte nicht zu einer weiteren Vergrößerung der Amplitude. In den bisherigen Untersuchungen wurde nur gesichert, dass der VCO für eine Tuningspannung von $V_{\text {tune }}=0 \mathrm{~V}$ eine stabile Schwingung aufweist. Eine Erhöhung der Tuningspannung führt aber aufgrund der spannungsabhängigen Varaktorkapazität zu einer Verkleinerung der resultierenden Tankkapazität und damit zu einer Vergrößerung der VCO-Frequenz. Da der äquivalente Tankwiderstand $R_{t}$ nach (20) frequenzabhängig ist führt eine Erhöhung der Frequenz zu einer Erhöhung dieses Widerstands. Nach Gleichung (30) ist die benötigte Weite $W_{n}$ invers proportional zu $R_{t}^{2}$. Daher ist sichergestellt, dass ein für eine Tuningspannung von $V_{\text {tune }}=0 \mathrm{~V}$ entworfener VCO auch für höhere Tuningspannungen eine stabile Schwingung aufweist. Der vorgestellte systematische Entwurfsprozess ermöglicht es die minimale Weite des dif- 


\begin{tabular}{|c|c|c|}
\hline Param. & Spez. & erreicht \\
\hline$f_{0}$ & $2.4 \mathrm{GHz}$ & $2.4 \mathrm{GHz}$ \\
\hline$V_{D D}$ & $2.5 \mathrm{~V}$ & $2.5 \mathrm{~V}$ \\
\hline$I_{\text {bias }}$ & $\leq 1 \mathrm{~mA}$ & $1 \mathrm{~mA}$ \\
\hline$T R$ & $\geq 20 \%$ & $34 \%$ \\
\hline$V_{t}$ & $\geq 1 \mathrm{~V}$ & $\geq 1.37 \mathrm{~V}$ \\
\hline$C_{\text {load }}$ & $\geq 100 \mathrm{fF}$ & $100 \mathrm{fF}$ \\
\hline \multicolumn{3}{|c|}{ Spule } \\
\hline Param. & Maple & Cadence \\
\hline $\mathrm{L}_{\mathrm{i}, \mathrm{p}}$ & $11.16 \mathrm{nH}$ & - \\
\hline $\mathrm{C}_{\mathrm{i}, \mathrm{p}}$ & $16.6 \mathrm{fF}$ & - \\
\hline $\mathrm{R}_{\mathrm{i}, \mathrm{p}}$ & $1824 \Omega$ & - \\
\hline
\end{tabular}

\begin{tabular}{|c|c|c|}
\hline \multicolumn{3}{|c|}{ Transistorpaar } \\
\hline Param. & Maple & Cadence \\
\hline$W_{n}$ & $>2.03 \mu \mathrm{m}$ & $>3.05 \mu \mathrm{m}$ \\
\hline $\mathrm{L}_{n}$ & $250 \mathrm{~nm}$ & $250 \mathrm{~nm}$ \\
\hline $\mathrm{R}_{0}$ & $10 \mathrm{k} \Omega$ & $9.84 \mathrm{k} \Omega$ \\
\hline $\mathrm{C}_{\text {NMOS }}$ & $3.97 \mathrm{fF}$ & $3.77 \mathrm{fF}$ \\
\hline \multicolumn{3}{|c|}{ Varaktor } \\
\hline Param. & Maple & Cadence \\
\hline$W_{v}$ & $150 \mu \mathrm{m}$ & $140 \mu \mathrm{m}$ \\
\hline $\mathrm{L}_{\mathrm{v}}$ & $500 \mathrm{~nm}$ & $500 \mathrm{~nm}$ \\
\hline$R_{v, p}$ & $3222 \Omega$ & - \\
\hline $\mathrm{C}_{\mathrm{v} 0}$ & $378 \mathrm{fF}$ & - \\
\hline
\end{tabular}

Abbildung 11. Spezifikation and Designparameter für $V_{\text {tune }}=0$.

ferentiellen Transistorpaares zu finden, bei der eine stabile Schwingung des VCOs garantiert ist. Dadurch wird der Effekt der Nichtlinearität des differentiellen Transistorpaares und damit der Einfluss der höheren Harmonischen minimiert. Zusätzlich erlaubt der Entwurfsprozess eine Optimierung der Ausgangsampliude des VCOs.

\section{Zusammenfassung}

Es wurde ein systematischer Entwurfsprozess für CMOS LC Tank VCOs basierend auf der Andronov-Hopf Bifurkationsanalyse und der nichtlinearen Modellierung der einzelnen Schaltungskomponenten gezeigt. Dieser alternative Ansatz erlaubt es verbesserte Starwerte zu erzeugen, die somit den Ausgangspunkt des Designs bilden. Dabei hat der Designer die Möglichkeit über Parametervariationen den Abstimmbereich und die Amplitude des VCOs mit Bezug auf geometrische Grundgrößen, wie Weiten und Längen der Transistoren, zu bestimmen beziehungsweise diese zu optimieren. Um die Anwendbarkeit der Methode zu verdeutlichen, wurde ein $2.4 \mathrm{GHz}$ VCO unter Verwendung eines $0.25 \mu \mathrm{m} \mathrm{RF}$ CMOS-Prozesses entworfen, der alle Spezifikationen erfüllt und zusätzlich eine hohe spektrale Reinheit besitzt.

\section{Literatur}

Hegazi, E. and Abidi, A. A.: Varactor Characteristics, Oscillator Tuning Curves and AM-FM Conversion, IEEE J. Solid-St. Circ., 38(6), 1033-1039, 2003.

Bremer, J., Zorn, C., and Mathis, W.:Bifurcation Analysis of an LCTank VCO Including the Variable Capacitance, Proc. 15th International Conference Mixed Design of Integrated Circuits and Systems, Poznan, Poland, 2008.

Bunch, R. L. and Raman, S.: Large-Signal Analysis of MOS Varactors in CMOS-Gm LC VCOs, IEEE J. Solid-St. Circ., 38(8), 1325-1332, 2003.
Buonomo, A. andLo Schiavo, A.: Modelling and analysis of differential VCOs, Int. J. Circ. Theor. App., 32, 117-131, 2004.

Enz, C., Krummenacher, F., and Vittoz, E.: An Analytical MOS Transistor Model Valid in All Regions of Operation and Dedicated to Low-Voltage and Low-Current Applications, Analog Integrated Circuits and Signal Processing, Kluwer, 8, 83-114, 1995.

Guckenheimer, J. and Holmes, P.: Nonlinear Oscillations, Dynamical systems and Bifurcations of Vector Fields, Springer Verlag, 178 pp., New York, 1983.

Hershenson, M., Hajimiri, A., Mohan, S., Boyd, S., and Lee, T.: Design and optimization of LC oscillators, Proceedings IEEE/ACM international conference on Computer-aided design, 65-69, 1999.

Hassard, B. D., Kazarinoff, N. D., and Wan, Y.-H.: Theory and Applications of Hopf Bifurcation, Cambridge University Press, Chapter 1 und 2, 1981.

Kirchgraber, U. and Stiefel, E.: Methoden der analytischen Störungsrechnung und ihre Anwendung, Teubner Verlag, Stuttgart, 21-30, 1978.

Kuznetsov, Y. A.: Elements of Applied Bifurcation Theory, Springer Verlag, New York, 3rd edn., 84-102, 2004.

Mathis, W.: Theorie nichtlinearer Netzwerke, Springer Verlag, Berlin, 375-379, 1987.

Mathis, W. and Russer, P.: Oscillator Design, in: Encyclopedia of RF and Microwave Engeneering, edited by: Chang, K., Wiley, 4, 3563-3589, 2005.

Prochaska, M., Belski, A., and Mathis, W.: Bifurcation Analysis of On-Chip Oscillators", Proc. IEEE International Symposium on Circuits and Systems (ISCAS), Cobe, Japan, 2005.

Tiebout, M.: Low-Power Low-Phase-Noise Differentially Tuned Quadrature VCO Design in Standard CMOS, IEEE J. Solid-St. Circ., 36(7), 1018-1024, 2001.

Yue, C. P. and Wong, S. S.: On-Chip Spiral Inductors with Patterned Ground Shields for Si-Based RF IC's, IEEE J. Solid-St. Circ., 33(5), 560-568, 1998.

Yue, C. P. and Wong, S. S.: Physical modeling of spiral inductors on silicon, IEEE T. Electron Dev., 47(3), 560-568, March 2000. 